Revue d'histoire de l'Amérique française

6. REVUE D.HISTOIRE DE L'AMÉRIQUE FRANÇAISE

\title{
Index des auteurs d'article ou de note de recherche
}

Index, 1987

Index des volumes XXXI-XL (juin 1977 - printemps 1987)

URI : https://id.erudit.org/iderudit/305684ar

DOI : https://doi.org/10.7202/305684ar

Aller au sommaire du numéro

Éditeur(s)

Institut d'histoire de l'Amérique française

ISSN

0035-2357 (imprimé)

1492-1383 (numérique)

Découvrir la revue

Citer ce document

(1987). Index des auteurs d'article ou de note de recherche. Revue d'histoire de l'Amérique française, 57-65. https://doi.org/10.7202/305684ar d'utilisation que vous pouvez consulter en ligne.

https://apropos.erudit.org/fr/usagers/politique-dutilisation/ 


\section{Index des auteurs d'article ou de note de recherche}


Certains articles sont signés par plusieurs auteurs. Consulter la liste des articles par titre pour la référence complète.

(NR) indique une note de recherche.

ALLAIRE, Gratien, Les engagements pour la traite des fourrures - évaluation de la documentation, 34, 1: 3-26

ALLAIRE, Gratien, Officiers et marchands: les sociétés de commerce des fourrures, 17151760, 40, 3: 409-428

ANGERS, François-Albert, Mesure de l'influence du chanoine Lionel Groulx sur son milieu, 32, 3: 357-384

ARÈS, Richard, Le père Joseph-Papin Archambault, $\mathbf{s}$. j. et l'École sociale populaire Témoignage -, 35, 4: 563-587

ARMSTRONG, Robert, L'industrie de l'amiante au Québec, 1878-1929, 33, 2: 187-195

BAILLARGEON, Denyse, La grève de Lachute (1947), 37, 2: 271-289

BATES, Réal, Les conceptions prénuptiales dans la vallée du Saint-Laurent avant 1725 (NR), 40, 2: 253-272

BATES, Réal, Les naissances illégitimes sur les rives du Saint-Laurent avant 1730 (NR), 40, 2: $239-252$

BEAUREGARD, Yves, Famille, parenté et colonisation en Nouvelle-France (NR), 39, 3: 391-405

BÉDARD, Marc-André, La présence protestante en Nouvelle-France, 31, 3: 325-349

BÉLANGER, Réal, Les députés conservateursnationalistes et le gouvernement Borden - le cas d'Albert Sévigny (1911-1913), 35, 1: 33-54

BeLLAVANCE, Claude, Financement et industrie en Mauricie, 1900-1950, 40, 1: 29-50

BELLAVANCE, Claude, Patronat et entreprise au XXe siècle: l'exemple mauricien, 38, 2: 181-201

BERGERON, Réjean, Les questions internationales dans les premiers inédits de Lionel Groulx (1895-1909), 34, 2: 245-255

BERNATCHEZ, Ginette, La société littéraire et historique de Québec (The Literary and Historical Society of Quebec) 1824-1890, 35, 2: $179-192$

BERNIER, Gérald, Appropriation foncière et bourgeoisie marchande: éléments pour une analyse de l'économie marchande du BasCanada avant 1846, 36, 2: 163-194

BERNIER, Gérald, Sur quelques effets de la rupture structurelle engendrée par la Conquête au Québec: 1760-1854, 35, 1: 69-95

BERNIER, Jacques, François Blanchet et le mouvement réformiste en médecine au début du XIXe siècle, 34, 2: 223-244

BERNIER, Jacques, La construction domiciliaire à Québec 1810-1820, 31, 4: 547-561

BERNIER, Jacques, La standardisation des études médicales et la consolidation de la profession dans la deuxième moitié du XIXe siècle, $37,1: 51-65$

BERTHIAUME, Pierre, Le tremblement de terre de 1663: les convulsions du verbe ou la mystification du logos chez Charlevoix, 36, 3: $375-387$

BERVIN, George, Aperçu sur le commerce et le crédit à Québec 1820-1830, 36, 4: 527-551

BERVIN, George, Les sources archivistiques: leur utilisation dans l'étude de la bourgeoisie marchande bas-canadienne (1800-1830), 38, 2: 203-222

BEUTLER, Corinne, Le rôle du blé à Montréal sous le Régime seigneurial, 36, 2: 241-262

BOUCHARD, Gérard, Introduction à l'étude de la société saguenayenne aux XIXe et XXe siècles, $31,1:$ 3-27

BOUCHARD, Gérard, La dynamique communautaire et l'évolution des sociétés rurales québécoises aux 19e et $20 \mathrm{e}$ siècles. Construction d'un modèle, $40,1: 51-71$

BOUCHARD, Gérard, Les notables du Saguenay au $20 \mathrm{e}$ siècle à travers deux corpus biographiques, 39, 1: 3-23

BOUCHARD, Gérard, Le projet d'histoire sociale de la population du Saguenay: l'appareil méthodologique, 32, 1: 41-56

BOUCHER, Phillp P., Comment se forme un ministre colonial. L'initiation de Colbert 1651-1664, 37, 3: 431-452

BOUCHER, Réal, L'endettement de l'évêché de Saint-Hyacinthe au XIXe siècle: le rôle décisif de Charles LaRocque dans l'extinction de cette dette, $33,4: 557-574$

BOURASSA, Joanne, Le rôle de Lionel Groulx comme éveilleur de conscience nationale de ses compatriotes (Concours Lionel-Groulx), 32, 3: 449-454

BRIĖRE, Jean-François, Le commerce triangulaire entre les ports terre-neuviers français, les pêcheries d'Amérique du Nord et Mar- 
seille au $18 \mathrm{e}$ siècle: nouvelles perspectives, 40, 2: 193-214

BRIÈRE, Jean-François, L'État et le commerce de la morue de Terre-Neuve en France au XVIIIe siècle, 36, 3: 323-338

BROUILLETTE, Normand, Financement et industrie en Mauricie, 1900-1950, 40, 1: 29-50

BROWN, Margaret Kimball, La colonisation française de l'Illinois: une réévaluation (NR), 39, 4: 583-591

BRUNET, Manon, Faire l'histoire de la littérature française du XIXe siècle québécois, 38 , 4: 523-547

BURGESS, Joanne, L'industrie de la chaussure à Montréal: 1840-1870 - Le passage de l'artisanat à la fabrique, 31, 2: 187-210

CELLARD, André, Ordre et désordre: le Montreal Lunatic Asylym et la naissance de l'asile au Québec, 39, 3: 345-367

CHARBONNEAU, Hubert, Colonisation, climat et âge au baptême des Canadiens au XVIIe siècle, 38, 3: 341-356

CHARBONNEAU, Hubert, Démographie différentielle en Nouvelle-France: villes et campagnes, 38, 3: 357-378

CHARBONNEAU, Hubert, Quelques comportements des Canadiens au XVIIe siècle d'après les registres paroissiaux, $31,1: 49$ 73

CHARLAND, Jean-Pierre, Le réseau d'enseignement public bas-canadien, 1841-1867: une institution de l'État libéral, 40, 4: 505535

CHOUINARD, Denis, La carrière politique de René Chaloult: l'art de promouvoir une politique nationaliste tout en sauvegardant son avenir politique, $39,1: 25-50$

CHOUINARD, Denis, Des contestataires pragmatiques: les Jeune-Canada, 1932-1938, 40, 1: $5-28$

CHOUINARD, Denys, Alfred Charpentier face au gouvernement du Québec, 1935-1946, 31, 2: 211-227

CLERMONT, Norman, Le gougou de Champlain et les croyances algonquiennes (NR), 35, 3: 377-381

CLICHE, Marie-Aimée, Les attitudes devant la mort d'après les clauses testamentaires dans le Gouvernement de Québec sous le Régime français, 32, 1: 57-94

CLICHE, Marie-Aimée, Les confréries dans le gouvernement de Québec sous le Régime français, 39, 4: 491-522
CODIGNOLA, Luca, L'Amérique du Nord et la Sacrée Congrégation «de Propaganda Fide», 1622-1799 - Guides et inventaires, 33, 2: 197-214

COMEAULT, Gilbert-L., La question des écoles du Manitoba - Un nouvel éclairage, 33, 1: 3-23

COUPAL, Jean-Paul, Les dix dernières années de la Revue d'histoire de l'Amérique française 1972-1981 (NR), 36, 4: 553-567

COURVILLE, Serge, Espace, territoire et culture en Nouvelle-France: une vision géographique, 37, 3: 417-429

COUTURE, Claude, La Conquête de 1760 et le problème de la transition au capitalisme, 39 , 3: $369-389$

CYR, Céline, Les alliances matrimoniales exogames dans le Gouvernement de Québec 1700-1760, 35, 1: 3-32

DE BONVILLE, Jean, La liberté de presse à la fin du XIXe siècle: le cas de Canada-Revue, 31, 4: 501-523

DE FRÉMINVILLE, Marine, Les origines des travailleurs de 1'Alcan au Saguenay, 19251939, 37, 2: 291-308

DEBIEN, Gabriel, Les engagés pour le Canada partis de Nantes (1725-1732) (NR), 33, 4: 583-586

DECHÊNE, Louise, La rente du faubourg SaintRoch à Québec - 1750-1850, 34, 4: 569-596

DESJARDINS, Pauline, La Coutume de Paris et la transmission des terres - Le rang de la Beauce à Calixa-Lavallée de 1730 à 1975 , 34, 3: 331-339

DESROCHERS, Luc, Les facteurs d'apparition du syndicalisme catholique dans l'imprimerie et les déterminants de la stratégie syndicale 1921-1945, 37, 2: 241-269

DESSUREAULT, Christian, L'égalitarisme paysan dans l'ancienne société rurale de la vallée du Saint-Laurent: éléments pour une ré-interprétation, 40, 3: 373-407

DICKINSON, John A., Annaotaha et Dollard vus de l'autre côté de la palissade, 35, 2: 163-178

DICKINSON, John A., La guerre iroquoise et la mortalité en Nouvelle-France 1608-1666, 36, 1: 31-54

DINEL, Guy, Les alliances matrimoniales exogạmes dans le Gouvernement de Québec 1700-1760, 35, 1: 3-32

DIONNE, Raoul, L'origine acadienne de Moncton: le Coude, 37, 3: 399-416 
DROLET, Yves, Les questions internationales dans les premiers inédits de Lionel Groulx (1895-1909), 34, 2: 245-255

DUBUC, Alfred, L'influence de l'école des Annales au Québec, 33, 3: 357-386

DUCHESNE, Raymond, La bibliothèque scientifique de l'abbé Léon Provancher, 34, 4: 535-556

DUCHESNE, Raymond, Historiographie des sciences et des techniques au Canada, 35, 2: 193-215

DUFOUR, Pierre, La construction navale à Québec, 1760-1825: sources inexplorées et nouvelles perspectives de recherches, 35,2 : 231-251

DUMONT-JOHNSON, Micheline, Des garderies au XIXe siècle: Les salles d'asile des Soeurs Grises à Montréal, 34, 1: 27-55

EID, Nadia F., Éducation et classes sociales: analyse de l'idéologie conservatrice - cléricale et petite bourgeoisie - au Québec au milieu du 19e siècle, 32, 2: 159-179

EID, Nadia F., Théories de la famille et rapports famille-pouvoirs dans le secteur éducatif au Québec et en France (1850-1960), 34, 2: 197-221

FALARDEAU, Jean-Charles, L'oeuvre de Guy Frégault, 35, 1: 55-68

FECTEAU, Jean-Marie, Régulation sociale et répression de la déviance au Bas-Canada au tournant du 19e siècle (1791-1815), 38, 4: 499-521

FERRETTI, Lucia, Mariage et cadre de vie familiale dans une paroisse ouvrière montréalaise: Sainte-Brigide, 1900-1914, 39, 2: 233-251

FERRON, Jean-Olivier, Qui perd sa langue, perd sa foi? (NR), 33, 2: 263-265

FOURNIER, Marcel, Édouard Montpetit et l'université morderne, ou l'échec d'une génération, 36, 1: 3-29

FRENETTE, Nicole LAURIN-, voir LAURINFRENETTE, Nicole

FRENETTE, Yves, Les éditoriaux de La Presse, 1934-1936: une défense de la démocratie libérale (NR), 33, 3: 451-462

GADOURY, Lorraine, Démographie différentielle en Nouvelle-France: villes et campagnes, 38, 3: 357-378

GAGNÉ, Suzanne, Lionel Groulx, historien d'hier ou d'aujourd'hui (Concours LionelGroulx), 32, 3: 455-458
GAGNON, Serge, Le milieu d'origine du clergé québécois 1775-1840: mythes et réalités, 37, 3: 373-397

GALARNEAU, France, L'élection partielle du quartier-ouest de Montréal en 1832: analyse politico-sociale, 32, 4: 565-584

GAUDREAU, Guy, Le rapport agriculture - forêt au Québec (NR), 33, 1: 67-78

GERMAIN, Annick, L'émergence d'une scène politique: mouvement ouvrier et mouvements de réforme urbaine à Montréal au tournant du siècle - Essai d'interprétation - , 37, 2: 185-199

GERmaIN, Pierre, Les récits de voyages de Pierre-Esprit Radisson - étude d'histoire bibliographique (NR), 34, 3: 407-414

GERVAIS, Gaétan, Le commerce de détail au Canada (1870-1880), 33, 4: 521-556

GOSSAGE, Peter, Les enfants abandonnés à Montréal au 19e siècle: la Crèche d'Youville des Soeurs Grises, 1820-1871, 40, 4: 537559

GOUDREAU, Serge, Famille, parenté et colonisation en Nouvelle-France (NR), 39, 3: 391405

GRANDBOIS, Maryse, Le développement des disparités régionales en Gaspésie 17601960, 36, 4: 483-506

GREENWOOD, F. Murray, L'insurrection appréhendée et l'administration de la justice au Canada: le point de vue d'un historien, 34, 1: 57-93

GREENWOOD, F. Murray, Les patriotes et le gouvernement responsable dans les années $1830,33,1: 25-37$

HAMEL, Thérèse, Obligation scolaire et travail des enfants au Québec: 1900-1950, 38, 1: $39-58$

HARDY, Jean-Pierre, Quelques aspects du niveau de richesse et de la vie matérielle des artisans de Québec et de Montréal, 1740$1755,40,3: 339-372$

HAREVEN, Tamara K., Les grands thèmes de l'histoire de la famille aux États-Unis, 39, 2 : 185-209

HEAP, Margaret, La grève des charretiers à Montréal, 1864, 31, 3: 371-395

HEAP, Ruby, Un chapitre dans l'histoire de l'éducation des adultes au Québec: les écoles du soir, 1889-1892, 34, 4: 597-625

HEAP, Ruby, La Ligue de l'Enseignement (1902-1904): héritage du passé et nouveaux défis, 36, 3: 339-373 
HÉROUX, Andrée, Famille, parenté et colonisation en Nouvelle-France (NR), 39, 3: 391405

HUOT, Christiane, Groulx éveilleur de conscience nationale (Concours Lionel-Groulx), 32, 3: 435-448

IGARTUA, José E., Le comportement démographique des marchands de Montréal vers 1760, 33, 3: 427-445

IGARTUA, José E., Les origines des travailleurs de l'Alcan au Saguenay, 1925-1939, 37, 2: 291-308

JARNOUX, Philippe, La colonisation de la seigneurie de Batiscan aux $17 \mathrm{e}$ et $18 \mathrm{e}$ siècles: l'espace et les hommes, 40, 2: 163-191

JAUMAIN, Serge, Paris devant l'opinion canadienne-française: les récits de voyages entre 1820 et $1914,38,4: 549-568$

JEAN, Dominique, Les parents québécois et l'État canadien au début du programme des allocations familiales: 1944-1955, 40, 1: 7395

JEAN, Michèle, Famille, parenté et colonisation en Nouvelle-France (NR), 39, 3: 391-405

JOHNSON, Micheline DUMONT-, voir DUMONT-JOHNSON, Micheline

JONES, Richard, La carrière politique de René Chaloult: l'art de promouvoir une politique nationaliste tout en sauvegardant son avenir politique, 39, 1: 25-50

KENNY, Stephen, Attitudes envers la langue française pendant les années 1840 (NR), 34, 2: $257-267$

KESTEMAN, Jean-Pierre, Les premiers journaux du district de Saint-François (18231845) (NR), 31, 2: 239-253

KESTEMAN, Jean-Pierre, Les travailleurs à la construction du chemin de fer dans la région de Sherbrooke (1851-1853), 31, 4: 525-545

KEYES, John, Un commis des trésoriers généraux de la marine à Québec - Nicolas Lanoullier de Boisclerc, 32, 2: 181-202

KEYES, John, La diversification de l'activité économique de Timothy Hibbard Dunn, commerçant de bois à Québec, 1850-1898, 35, 3: 323-336

KOLISH, Evelyn, Le Conseil législatif et les bureaux d'enregistrement (1836), 35, 2: 217-230

KOLISH, Evelyn, L'introduction de la faillite au Bas-Canada: conflit social ou national?, 40, 2: $215-235$
KONDERT, Reinhart, Les Allemands en Louisiane de 1721 à 1732, 33, 1: 51-65

LABERGE, Alain, État, entrepreneurs, habitants et monopole: le «privilège» de la pêche au marsouin dans le Bas Saint-Laurent 17001730, 37, 4: 543-555

LABERGE, Alain, Famille, parenté et colonisation en Nouvelle-France (NR), 39, 3: 391405

LACHANCE, André, Une étude de mentalité: les injures verbales au Canada au XVIIIe siècle (1712-1748) (NR), 31, 2: 229-238

LACHANCE, Paul, L'effet du déséquilibre des sexes sur le comportement matrimonial: comparaison entre la Nouvelle-France, Saint-Domingue et la Nouvelle-Orléans, 39, 2: $211-231$

LACROIX, Benoît, Lionel Groulx, cet inconnu?, 32, 3: 325-346

LAFRANCE, Marc, Art militaire et technique de guerre: le Fort de Chambly de 1710-1711, 37, 1: 21-49

LALONDE, André-N., L'intelligentsia du Québec et la migration des Canadiens français vers l'Ouest canadien, 1870-1930, 33, 2: 163-185

LAMARRE, Jean, Du Québec vers les ÉtatsUnis: l'étude des lieux d'origine (NR), 38 , 3: $409-422$

LAMOUREUX, Diane, La lutte pour le droit à l'avortement (1969-1981), 37, 1: 81-90

LANDRY, Yves, Démographie différentielle en Nouvelle-France: villes et campagnes, 38, 3: 357-378

LANDRY, Yves, Le registre de population de la Nouvelle-France: un outil pratique au service de la démographie historique et de l'histoire sociale (NR), 38, 3: 423-426

LANDRY, Yves, Quelques comportements des Canadiens au XVIIe siècle d'après les registres paroissiaux, 31, 1: 49-73

LANTHIER, Pierre, Financement et industrie en Mauricie, 1900-1950, 40, 1: 29-50

LANTHIER, Pierre, Stratégie industrielle et développement régional: le cas de la Mauricie au XXe siècle, 37, 1: 3-19

LAPERRIÈRE, Guy, «Persécution et exil»: la venue au Québec des congrégations françaises, 1900-1914, 36, 3: 389-411

LAPIERRE, André, Le manuel de l'abbé Thomas Maguire et la langue québécoise au XIXe siècle, 35, 3: 337-354 
LAPOINTE, Michelle, Le syndicat catholique des allumettières de Hull, 1919-1924, 32, 4: 603- 628

LAURENCE, Gérard, Le début des affaires publiques à la télévision québécoise 1952 1957, 36, 2: 213-239

LAURIN-FRENETTE, Nicole, Théories de la famille et rapports famille-pouvoirs dans le secteur éducatif au Québec et en France (1850-1960), 34, 2: 197-221

LAVOIE, Yolande, Le projet d'histoire sociale de la population du Saguenay: l'appareil méthodologique, 32, 1: 41-56

LEBEL-GAGNON, Louise, Le milieu d'origine du clergé québécois 1775-1840: mythes et réalités, 37, 3: 373-397

LEBLANC, Robert G., Colonisation et rapatriement au Lac-Saint-Jean (1895-1905), 38, 3: 379-408

LESSARD, Rénald, Famille, parenté et colonisation en Nouvelle-France (NR), 39, 3: 391405

LÉVESQUE, Andrée, Mères ou malades: les Québécoises de l'entre-deux-guerres vues par les médecins, 38, 1: 23-37

LÉVESQUE, Andrée, Le Québec et le monde communiste: Cowansville 1931, 34, 2: 171182

LIEBEL, Jean, On a vieilli Champlain, 32, 2: 229-237

LITALIEN, Raymonde, Jean-Baptiste-Nicolas Roch de Ramezay et sa famille, au Canada et en France, après 1760 (NR), 37, 4: 603610

LITTLE, Jack I., Les investisseurs français et le nationalisme canadien-français. La Compagnie de colonisation et de crédit des Cantons de l'Est - 1881-1893, 32, 1: 19-39

MANN TROFIMENKOFF, Susan, voir TROFIMENKOFF, Susan Mann

MARKOWSKI, France, Les notables du Saguenay au $20 \mathrm{e}$ siècle à travers deux corpus biographiques, 39, 1: 3-23

MARMIER, Jean, Le Récit de M. de Courcelles au lac Ontario (1671), et Dollier de Casson (NR), 32, 2: 239-250

MARTIN, Richard, Le congrès des métiers et du travail et la guerre civile espagnole (NR), 33, 4: 575-581

MATHIEU, Jacques, Les alliances matrimoniales exogames dans le Gouvernement de Québec 1700-1760, 35, 1: 3-32
MATHIEU, Jacques, Hommage à Lionel Groulx, 32, 3: 323

MICHEL, Louis, Un marchand rural en Nouvelle-France - François-Augustin Bailly de Messein, 1709-1771, 33, 2: 215-262

MIMEAULT, Mario, Les entreprises de pêche à la morue de Joseph Cadet 1751-1758, 37, 4: 557-572

MONTPETIT, Raymond, Un exemple de peinture d'histoire au Québec: Charles Huot à l'Assemblée nationale (NR), 31, 3: 397-405

MORIN, Yvan, La représentativité de l'inventaire après décès - l'étude d'un cas: Québec au début du XIXe siècle, 34, 4: 515-533

MORTON, Desmond, E. P. Thompson dans des arpents de neige: les historiens canadiensanglais et la classe ouvrière, 37,2 : 163-184

NADON, Dominique, Ordre et désordre: le Montreal Lunatic Asylum et la naissance de l'asile au Québec, 39, 3: 345-367

NOEL, Françoise, La gestion des seigneuries de Gabriel Christie dans la vallée du Richelieu (1760-1845), 40, 4: 561-582

NOEL, Johanne, Famille, parenté et colonisation en Nouvelle-France (NR), 39, 3: 391405

OTIS, Yves, Les notables du Saguenay au 20e siècle à travers deux corpus biographiques, 39, 1: 3-23

OUELLET, Fernand, Les classes dominantes au Québec, 1760-1840. Bilan historiographique, 38, 2: 223-243

PAQUET, Gilles, Pour une méso-histoire du XIXe siècle canadien, 33, 3: 387:425

PAQUET, Gilles, Stratégie foncière de l'habitant: Québec (1790-1835), 39, 4: 551-581

PAQUET, Gilles, Sur quelques discontinuités dans l'expérience socio-économique du Québec: une hypothèse, 35, 4: 483-521

PAQUET, Lucie, Famille, parenté et colonisation en Nouvelle-France (NR), 39, 3: 391405

PAQUETTE, Lyne, Les naissances illégitimes sur les rives du Saint-Laurent avant 1730 (NR), 40, 2: 239-252

PAQUETTE, Plerre, Industries et politiques minières au Québec: une analyse économique 1896-1975, 37, 4: 573-602

PARISEAU, Jean, Les mouvements sociaux, la violence et les interventions armées au Québec 1867-1967, 37, 1: 67-79 
PARMENTIER, Francis, Réception de La Lanterne par la presse canadienne-française (NR), 34, 2: 269-274

PÉNISSON, Bernard, Le Commissariat canadien à Paris (1882-1928), 34, 3: 357-376

PETIT, Françoise-Eugénie, Aménagement rural et maîtrise de l'espace au XXe siècle par les collectivités paysannes des régions défavorisées en France et au Québec (NR), 35, 2: 253-261

PIERRE-DESCHÊNES, Claudine, Santé publique et organisation de la profession médicale au Québec 1870-1918, 35, 3: 355-375

PLAMONDON, Lilianne, Une femme d'affaires en Nouvelle-France: Marie-Anne Barbel, veuve Fornel, 31, 2: 165-185

PORTER, John R., Un projet de musée national à Québec à l'époque du peintre Joseph Légaré (1833-1853) (NR), 31, 1: 75-82

PORTES, Jacques, «La Capricieuse» au Canada, 31, 3: 351-370

POTHIER, Louisette, Un patriote inconnu: Joseph-Édouard Turcotte (NR), 38, 1: 59-71

POUYEZ, Christian, Un inventaire descriptif des documents de la session, 1841-1936 (NR), 31, 4: 563-574

POZZO, Jeannine, Les alliances matrimoniales exogames dans le Gouvernement de Québec 1700-1760, 35, 1: 3-32

PROULX, Gilles, Le dernier effort de la France au Canada - secours ou fraude? -, 36, 3: 413-426

PROULX, Gilles, Soldat à Québec, 1748-1759, 32, 4: 535-563

PROVOST, Guy, Conception de la littérature chez Joseph-Sabin Raymond, 32, 4: 585 602

RAMIREZ, Bruno, Du Québec vers les ÉtatsUnis: l'étude des lieux d'origine (NR), 38, 3: 409-422

RÉMILLARD, Juliette, Lionel Groulx: bibliographie (1964-1979), 32, 3: 465-523

ROBERT, Jean-Claude, Urbanisation et population: le cas de Montréal en 1861, 35, 4: 523-535

ROBERT, Lucie, Camille Roy et Lionel Groulx: la querelle de L'appel de la race, 32, 3: 399-405

ROUILLARD, Jacques, Implantation et expansion de l'Union internationale des travailleurs en chaussures au Québec de 1900 à 1940, 36, 1: 75-105
ROUILLARD, Jacques, Le militantisme des travailleurs au Québec et en Ontario, niveau de syndicalisation et mouvement de grève (1900-1980), 37, 2: 201-225

ROUILLARD, Jacques, Mutations de la Confédération des travailleurs catholiques du Canada (1940-1960), 34, 3: 377-405

ROUSSEAU, François, Hôpital et société en Nouvelle-France: l'Hôtel-Dieu de Québec à la fin du XVIIe siècle, 31, 1: 29-47

ROY, Jean, Le clergé nicolétain, 1885-1904: aspects sociographiques (NR), 35, 3: 383395

ROY, Raymond, Quelques comportements des Canadiens au XVIIe siècle d'après les registres paroissiaux, 31, 1: 49-73

RUDIN, Ronald, Naissance et déclin d'une élite locale: la Banque des Cantons de l'Est, 1859-1912, 38, 2: 165-179

ST-AMANT, Jean-Claude, La propagande de l'École sociale populaire en faveur du syndicalisme catholique 1911-1949, 32, 2: 203 228

ST-GEORGES, Lise, Commerce, crédit et transactions foncières: pratiques de la communauté marchande du bourg de L'Assomption, 1748-1791, 39, 3: 323-343

ST-PIERRE, Jacques, Les alliances matrimoniales exogames dans le Gouvernement de Québec 1700-1760, 35, 1: 3-32

SALÉE, Daniel, Appropriation foncière et bourgeoisie marchande: éléments pour une analyse de l'économie marchande du BasCanada avant 1846, 36, 2: 163-194

SÉGALEN, Martine, Sous les feux croisés de l'histoire et de l'anthropologie: la famille en Europe, 39, 2: 163-184

SÉGUIN, Normand, L'agriculture de la Mauricie et du Québec 1850-1950, 35, 4: 537-562

SÉNÉCAL, André, La thèse messianique et les Franco-Américains, 34, 4: 557-567

SÉVIGNY, P.-André, Le commerce du blé et la navigation dans le Bas-Richelieu avant $1849,38,1: 5-21$

SIROIS, Jean, Le chanoine Groulx, historien d'hier et d'aujourd'hui (Concours LionelGroulx), 32, 3: 459-464

STANLEY, George F.G., Le projet Riel (NR), 33, 3: $447-450$

TÉTREAULT, Martin, Les maladies de la misère: aspects de la santé publique à Montréal $1880-1914,36$, 4: 507-526 
THÉRIAULT, Serge A., Dominique-Marie Varlet - de l'Église de Québec à la réforme d'Utrecht, 36, 2: 195-212

THIVIERGE, Marîse, Les artisans du cuir à Québec (1660-1760), 34, 3: 341-356

TOUSIGNANT, Pierre, Groulx et l'histoire: interrogation sur le passé en vue d'une direction d'avenir, 32, 3: 347-356

TREMBLAY, Robert, La formation matérielle de la classe ouvrière à Montréal entre 1790 et $1830,33,1: 39-50$

TREMBLAY, Robert, La grève des ouvriers de la construction navale à Québec (1840), 37, 2: $227-239$

TRÉPANIER, Lise, Rameau de Saint-Père et le métier d'historien, 33, 3: 331-355

TRÉPANIER, Pierre, Rameau de Saint-Père et le métier d'historien, 33, 3: 331-355

TRÉPANIER, Plerre, Du système colonial des peuples modernes: un inédit de Rameau de Saint-Père, 36, 1: 55-74

TRIFIRO, Luigi, Une intervention à Rome dans la lutte pour le suffrage féminin au Québec (1922), 32, 1: 3-18

TROFIMENKOFF, Susan Mann, Les femmes dans l'oeuvre de Groulx, 32, 3: 385-398

TRUDEL, François, Les Inuit du Labrador méridional face à l'exploitation canadienne et française des pêcheries (1700-1760), 31, 4: 481-499
TRUDEL, Marcel, Jean Nicollet dans le Lac Supérieur et non dans le Lac Michigan, 34, 2: 183-196

TURGEON, Laurier, Pour redécouvrir notre $16 \mathrm{e}$ siècle: les pêches à Terre-Neuve d'après les archives notariales de Bordeaux, 39, 4: 523549

VAILLANCOURT, François, Qui perd sa langue, perd sa foi? (NR), 33, 2: 263-265

VERRETTE, Michel, L'alphabétisation de la population de la ville de Québec de 1750 à 1849, 39, 1: 51-76

VOISINE, Nive, Rome et le Canada: la mission de Mgr Conroy, 33, 4: 499-519

WALLOT, Jean-Pierre, A la recherche de la nation: Maurice Séguin (1918-1984), 38, 4: 569-590

WALLOT, Jean-Pierre, Groulx historiographe, 32, 3: 407-433

WALLOT, Jean-Pierre, L'Histoire et la recherche du sens. Discours de réception à l'Académie canadienne-française, 37, 4: 533-542

WALLOT, Jean-Pierre, Pour une méso-histoire du XIXe siècle canadien, 33, 3: 387-425

WALLOT, Jean-Pierre, Stratégie foncière de l'habitant: Québec (1790-1835), 39, 4: 551581

WALLOT, Jean-Pierre, Sur quelques discontinuités dans l'expérience socio-économique du Québec: une hypothèse, 35, 4: 483-521 